Document downloaded from:

http://hdl.handle.net/10251/118179

This paper must be cited as:

Benalcazar-Parra, C.; Ye Lin, Y.; Garcia Casado, J.; Monfort-Orti, R.; Alberola Rubio, J.; Perales Marín, AJ.; Prats-Boluda, G. (2018). Electrohysterographic characterization of the uterine myoelectrical response to labor induction drugs. Medical Engineering \& Physics. 56:27-35. https://doi.org/10.1016/j.medengphy.2018.04.002

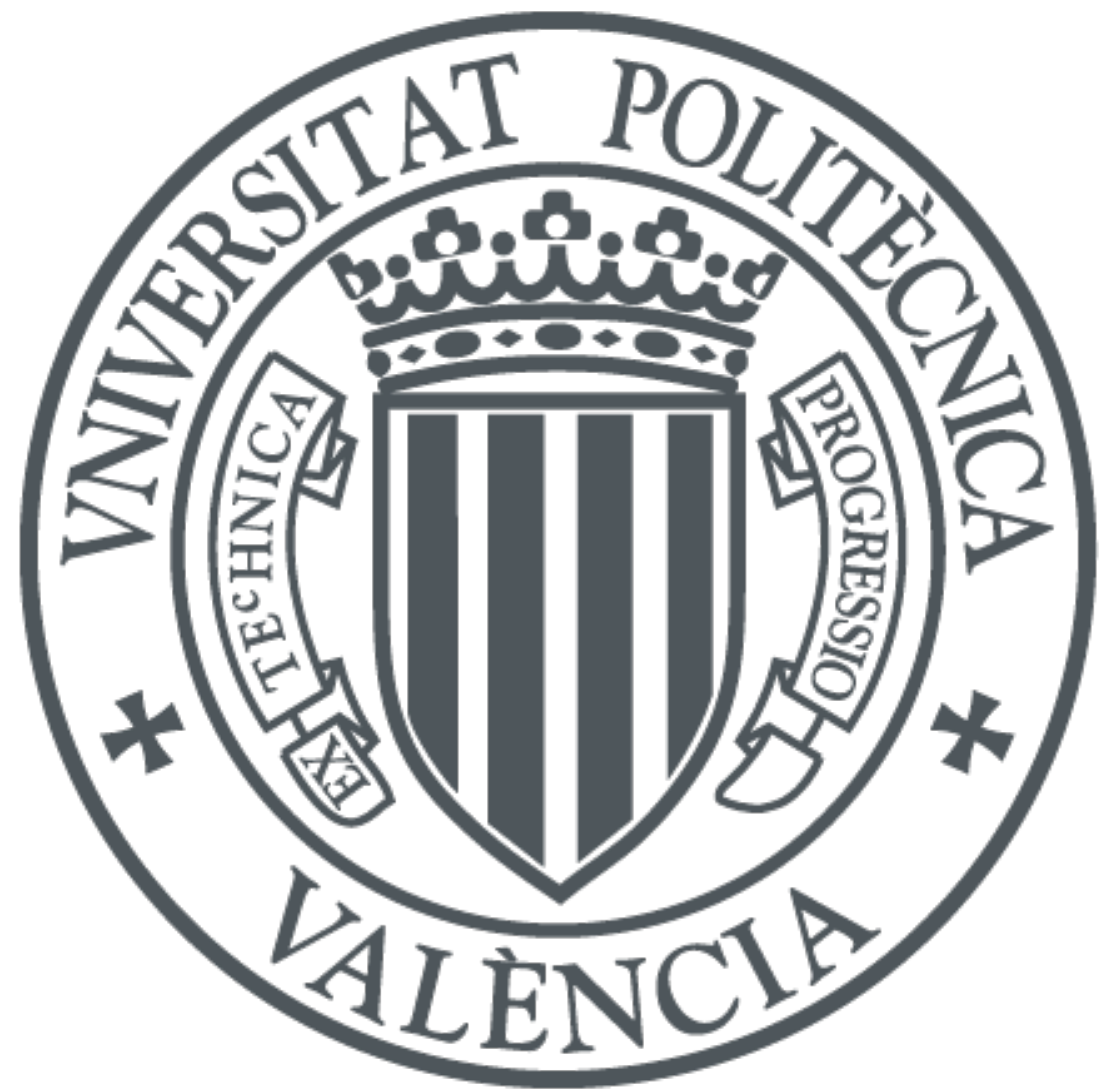

The final publication is available at

http://doi.org/10.1016/j.medengphy.2018.04.002

Copyright Elsevier

Additional Information 
Title:

\section{Electrohysterographic characterization of the uterine 3 myoelectrical response to labor induction drugs}

4 Running heads:

$5 \quad$ Uterine myoelectrical response to labor induction drugs

6 Authors:

7 Carlos Benalcazar-Parra MSc ${ }^{\mathrm{a}}$, Yiyao Ye-Lin $\mathrm{Phd}^{\mathrm{a}}$, Javier Garcia-Casado $\mathrm{PhD}^{\mathrm{a},{ }^{*}}$, Rogelio Monfort-

8 Orti ${ }^{\mathrm{b}}$, Jose Alberola-Rubio $\mathrm{PhD}^{\mathrm{b}}$, Alfredo Perales $\mathrm{PhD}^{\mathrm{b}}$ and Gema Prats-Boluda $\mathrm{PhD}^{\mathrm{a}}$

$9 \quad$ Affiliations

10 a. Centro de Investigación e Innovación en Bioingeniería, Universitat Politècnica de València, Edif.

11 8B, Camino de Vera SN, 46022 Valencia, Spain. \{carbepar, yiye, jgarciac,gprats\}@ci2b.upv.es

12 b. Servicio de Obstetricia y Ginecología, Hospital Universitario y Politécnico La Fe de Valencia,

13 Valencia, Spain.roger1487@hotmail.com, palberola.rubio@gmail.com, perales_alf@gva.es

14 *Corresponding author:

15 Full name: Gema Prats Boluda

16 E-mail address: gprats@ci2b.upv.es

17 Full postal address: Centro de Investigación e Innovación en Bioingeniería, Universitat

18 Politècnica de Valéncia, Edif. 8B, Camino de Vera SN, 46022 Valencia, Spain

19 Telephone: +34963877007 ext. 76027

20

21

\section{Conflicts of Interest statement}

In accordance with my ethical obligation as a researcher, I am reporting that this research has received funding from Bial S.A. a company that may be affected by the research reported in the enclosed paper.

It has also been funded by the Spanish Ministry of Economy and Competitiveness and the European Regional Development Fund (DPI2015-68397-R) 


\section{Abstract}

Labor induction is a common practice to promote uterine contractions and labor onset. Uterine electrohysterogram (EHG) has proved its suitability for characterizing the uterus electrophysiological condition in women with spontaneous labor. The aim of this study was to characterize and compare uterine myoelectrical activity during the first $4 \mathrm{~h}$ in response to labor induction drugs, Misoprostol (G1) and Dinoprostone (G2), by analyzing the differences between women who achieved active phase of labor and those who did not (successful and failed inductions). A set of temporal, spectral and complexity parameters were computed from the EHGbursts. As for successful inductions, statistical significant and sustained increases with respect to basal period were obtained for EHG amplitude, mean frequency, uterine activity index (UAI) and Teager, after 60' for the G1 group; duration, amplitude, number of contractions and UAI for the G2 group, after 120'. Moreover, Teager showed statistical significant and sustained differences between successful and failed inductions $\left(1.43 \pm 1.45 \mu \mathrm{V}^{2} \cdot \mathrm{Hz}^{2} \cdot 10^{5}\right.$ vs. $0.40 \pm 0.26 \mu \mathrm{V}^{2} \cdot \mathrm{Hz}^{2} \cdot 10^{5}$ after 240') for the G1 group, but not in the G2 group, probably due to the slower pharmacokinetics of this drug. These results revealed that EHG could be useful for successful induction prediction in the early stages of induction, especially when using Misoprostol.

\section{Keywords:}

Labor induction; Cervical Ripening; Drug effects; Surface Electromyography; Electrohysterography; Signal processing; Computer-assisted signal interpretation

\section{Introduction}

Labor induction is now a daily obstetric routine and is performed in an ever-increasing number of cases. It is indicated when the risk to mother and fetus of continuing the pregnancy outweighs that of terminating it. The goal is to achieve vaginal delivery by stimulating uterine contractions before the spontaneous onset of labor by promoting uterine contractions. Its use has increased significantly in recent years, from $9.0 \%$ of all births in 1989 to $23 \%$ in 2012 in the United States [1]. Labor induction can last for many hours (approximately 17-20 [2]), sometimes more than 36 hours, and in most cases does not ensure vaginal delivery. Indeed, almost $20 \%$ of all labor induction cases end in caesarean sections [3]. Predicting the success of induction is a key aspect in improving maternal and fetal well-being and reducing healthcare costs. The most common method of predicting success is based on cervix assessment by the Bishop score [4], although this method is subjective with poor reproducibility [5]. Other obstetric variables have been used for this purpose, such as cervical length, maternal age, height, weight, parity, and birth weight [6-10]. Some of the predictive capacity values given in the literature are in the area under the curve (AUC) of the receiver operating characteristic (ROC) curves ( 0.689 for cervical length and 0.72 for cervical dilatation [8]). The Bishop score and cervical length were found to achieve an AUC of 0.39 and 0.69 , respectively in [7] and Prado et al (2016) found an $\mathrm{AUC}=0.60$ for fetal weight. Consequently, no reliable models are available to predict the outcome of labor induction in clinical practice with common obstetric data.

Monitoring uterine activity is also fundamental to determining the uterine response to induction drugs, assessing maternal and fetal wellbeing and estimating the success of labor induction. In clinical settings, evaluating uterine dynamics can help clinicians to estimate the progress of labor 
and its outcome. Measuring intrauterine pressure (IUP) is the gold standard of uterine dynamics monitoring. Although this technique provides a reliable measure, it is somewhat limited due to its invasiveness, requiring the rupture of membranes [11]. The most commonly used method of noninvasively monitoring uterine activity consists of placing a tocodynamometer (TOCO) on the mother's abdomen [12]. However, this method does not provide reliable information, often experiences loss of contractions [12], repositioning the sensor may be required and its interpretation is highly dependent on the examiner's subjectivity .

Electrohysterography (EHG), the recording of uterine muscle electrical activity at the abdominal surface, has emerged as an alternative technique for non-invasive monitoring of uterine dynamics. EHG consists of intermittent bursts of action potentials associated with uterine contractions and basal activity when the uterus is at rest. In the literature, EHG has been widely used for monitoring uterine dynamics by generating TOCO-like and IUP-like signals [13-15], to differentiate between labor and non-labor contractions [16-18] and term vs preterm deliveries during pregnancy $[17,19,20]$. Figure 1 shows the main applications of EHG, at the research level, and the different type of parameters employed in each case. However, few studies focus on the uterine myoelectrical activity response to labor induction drugs [21,22]. In these latter, the uterine EMG signal characterization is based mainly on signal amplitude and only Toth et al [22] examined the possibility of predicting the success of labor induction. The question is still unclear of whether or not the distribution of spectral content and complexity parameters from the EHG signal undergo changes throughout labor induction by drugs (prostaglandins), and if these parameters -derived from the EHG-burst -might also be used to develop tools to predict successful induction.

The aim of the present work was thus to characterize and compare the uterine myoelectrical response of mothers administered Misoprostol and Dinoprostone, two commonly used labor induction drugs, by analyzing the modifications to uterine dynamics in the first hours of the induction process. For this, temporal, spectral and complexity parameters from EHG-burst were calculated and a set of parameters was identified capable of distinguishing between successful and unsuccessful inductions.

\section{Materials and methods}

\subsection{Signal acquisition}

Seventy-two recording sessions were conducted on expectant mothers with late term pregnancies admitted for cervical ripening and labor induction at the Hospital Universitario y Politécnico La Fe, in Valencia, Spain. The study adhered to the Declaration of Helsinki and was approved by the local medical ethical board. The subjects were informed of the nature of the study and provided written informed consent. The collected obstetric data included maternal age, body mass index (BMI), parity, gestations, pre-induction Bishop score and time to achieve active labor period (Table 1).

The recording sessions were divided into two groups according to the drug administered for labor induction: Group 1 (G1) were given a vaginal insert of $25 \mu \mathrm{g}$ of Misoprostol (Misofar, Bial, Coronado, Portugal) with repeated doses of up to four administrations every 4 hours. Group 2 (G2) was given a $10 \mathrm{mg}$ vaginal Dinoprostone insert (Propess, Ferring, Germany) in a single dose. The success of induction has been defined in the bibliography in different ways: vaginal delivery within 24 hours of induction [23,24], vaginal delivery within 48 hours of induction [25] and vaginal 
delivery at any time after induction [26]. However, when it is intended to assess the drug's success in activating uterine dynamics, regardless of whether or not the induction ended in a vaginal delivery, it can be considered successful if the mother reaches the active phase of labor. Both groups were therefore subdivided into induction success and failure groups, according to whether active labor was or was not achieved: G1S (Misoprostol-success), G1F (Misoprostol-failure), G2S (Dinoprostone-success), G2F (Dinoprostone-failure).

The recording sessions comprised 30 minutes of basal activity (before drug administration) and 4 hours after drug administration. For the EHG recordings, firstly the abdominal surface was carefully prepared with exfoliating gel (Nuprep, Weaver and Company, USA) to reduce skin-electrode impedance. Four monopolar disposable $\mathrm{Ag} / \mathrm{AgCl}$ electrodes (3M red dot 2560) were then placed on the abdominal surface (Figure 2): 2 electrodes (M1 and M2) were placed supraumbilically at each side of the median axis at $8 \mathrm{~cm}$ interelectrode distance to obtain one bipolar signal. A reference electrode was placed on the right hip and 1 ground electrode on the left hip. The electrodes were connected to a commercial biosignal amplifier (Grass 15LT+4 Grass 15A94; Grass Instruments, West Warwick, RI) to amplify and filter EHG signals between [0.1, 30] Hz. The signals were digitalized at a sampling frequency of $1000 \mathrm{~Hz}$. To eliminate low- and high-frequency interference and noise, the signals were additionally bandpass digitally filtered between $0.2-4 \mathrm{~Hz}$ and afterwards down-sampled at $20 \mathrm{~Hz}$ to reduce the amount of data and computational cost, giving rise to M1P and M2P preprocessed signals. TOCO signals were also recorded at the same time by a Corometrics 250cx (General Electric Healthcare) commercial maternal monitor, which sent the data to a PC at a sampling rate of $4 \mathrm{~Hz}$. Both the EHG and TOCO signals were stored for subsequent analysis.

\subsection{Data analysis}

A bipolar EHG signal (M1P-M2P) was first computed to reduce common mode interference. EHGbursts associated with uterine contractions were then manually segmented in the bipolar signal using the following criteria: significant increase in amplitude and/or frequency in comparison to rest activity, EHG-burst with a minimum duration of 30 seconds with no evidence of artifacts.

To characterize the uterine myoelectrical uterine state, a set of temporal, spectral and complexity parameters were computed from EHG-bursts. Uterine dynamics is clinically monitored using tocographic techniques, which consist of measuring the increase in pressure resulting from the shortening of uterine muscle fibers during contraction that provide information on the amplitude, duration and frequency of uterine contractions. The temporal parameters duration, peak to peak amplitude and number of contractions (NCT) were thus extracted every 30 minute, since this information is more familiar to obstetricians in labor management.

On the other hand, EHG-bursts are mainly composed of two distinct frequency components: -fast wave low (FWL), a low frequency component associated with EHG propagation and -fast wave high (FWH), a high frequency component related to uterine cells excitability [27]. The EHG bandwidth associated with these components mainly distributes between 0.2 to $1 \mathrm{~Hz}$ [13], although some authors consider that it can extend up to $4 \mathrm{~Hz}$ [28]. Many studies leave out the FWL and focus on the 0.34 to $1 \mathrm{~Hz}$ bandwidth, to minimize breathing and cardiac interferences [13]. It has also been shown that EHG-burst spectral content shifts to higher frequencies as labor approaches, in the 
range of 0.34 to $1 \mathrm{~Hz}$ [29]. Therefore, the following spectral parameters were obtained from the power spectral density distribution (PSD) of the EHG-bursts estimated by the periodogram method: mean frequency in the range $0.2-1 \mathrm{~Hz}(\mathrm{MF})$ and ratio between the energy content in high $(0.34-1$ $\mathrm{Hz}$ ) and low $(0.2-0.34 \mathrm{~Hz})$ frequency bands $(\mathrm{H} / \mathrm{L}$ ratio).

Since effective contractions, i.e. those leading to labor, require higher EHG-burst frequency content and amplitude, two other parameters that combine information in both the time and frequency domains were also computed: the Teager energy operator [30] and a novel uterine activity index (UAI), the latter defined as follows:

$$
U A I=\frac{\text { App } \cdot H / L \text { ratio }}{\text { duration }}
$$

Duration is used to describe UAI to emphasize the evolution of this parameter, as EHG-burst duration is expected to reduce as labor approaches [31]. Higher values of both the Teager and UAI parameters are considered to be associated with effective contractions.

As delivery approaches, myoelectrical activity becomes more predictable and less complex, some authors have proposed the use of nonlinear analysis to characterize EHG signals [28,32-34]. Sample entropy has been shown to be a promising parameter to discriminate between preterm and term labors [28] and also to assess the progress of labor [34]. As for Lempel-Ziv, Lemancewicz et al 2016 found a significant difference in its value in patients who gave preterm birth in less/more than 7 days. In this work, the parameters that measure the signal complexity of the EHG-burst were calculated in the $0.34-4 \mathrm{~Hz}$ bandwidth, as suggested in [28]. The parameters calculated were: sample entropy (SampEn), pattern length being $\mathrm{m}=2$ and tolerance $\mathrm{r}=0.15[28,35]$ and binary Lempel-Ziv complexity (LZ) [36].

Once EHG parameters were worked out in each EHG-Burst, the median values of each parameter were computed for the EHG-bursts present in the 30-minute analysis window. Subsequently, the mean and standard deviation of each parameter in each analysis window was calculated for all the women in each group.

Finally, statistical tests with different null and alternative hypothesis were performed by the MannWhitney test $(\alpha=0.05)$ to determine whether there were any significant changes in the EHG parameters in the first 4 hours of induction. First the analysis windows after drug administration were compared with basal activity: for the EHG characteristics that show growing tendencies as the induction of labor progresses, the null hypothesis indicates that the median of EHG characteristic is higher than that of the basal period; for EHG characteristics that show decreasing tendencies the null hypothesis indicates that the median of EHG characteristic is lower than that of the basal period. Statistical differences between successful and failed inductions (G1S vs G1F and G2S vs G2F), and between successful induction with different drugs (G1S vs G2S) were studied in a similar way to those defined before, i.e. when median values of a group were greater or lower than those of the other group. 


\section{Results}

2 A total of 72 subjects with singleton pregnancies were enrolled in the study, of which 35 received 3 Misoprostol and 37 Dinoprostone. The obstetric characteristics and success rate of both groups (G1 4 and G2) are summarized in Table 1. No statistically significant differences were found in the 5 obstetric data between both groups (maternal age, BMI, parity, gestation, rates of vaginal deliveries, rate of successful induction, time to achieve active labor period, neonatal outcomes such as arterial $\mathrm{PH}$ and vein $\mathrm{PH}$ ), except in the case of the Bishop score, which was significantly lower in G1 than $\mathrm{G} 2(\mathrm{p}=0.011)$, which means that onset conditions of labor induction were more unfavorable for the former group.

Figure 3A shows a representative EHG recording from a subject administered Misoprostol, who reached active labor. EHG-bursts after 4 hours from induction onset were of higher frequency and amplitude and lower duration than those of the basal period. Figure 3B shows a representative EHG recording of a subject induced by Dinoprostone, who also reached active labor. In this case, no notable changes were observed between the EHG-bursts of the last recording period, after 4 hours from induction onset, and those of the basal period, except for a slight increase in amplitude.

\subsection{Uterine myoelectrical response to Misoprostol induction drug}

Figure 4 shows mean values of the computed parameters for the EHG-bursts present in the 30minute analysis blocks for G1S and G1F (Misoprostol groups). In the former, EHG-burst duration decreased slightly and progressively and in the latter group showed no clear trend. Regarding EHGburst amplitude, G1S exhibited higher uterine contraction intensity than G1F in every $30 \mathrm{~m}$ period, with a sustained upward tendency throughout the recording session, from values of $159.4 \pm 47.8 \mu \mathrm{V}$ in the basal period to $245.8 \pm 83.9 \mu \mathrm{V}$ in the last $30 \mathrm{~m}$ period. Changes in EHG-burst amplitude were less noticeable in the G1F group (from $126.0 \pm 17.5 \mu \mathrm{V}$ to $142.0 \pm 19.9 \mu \mathrm{V}$ ). NCT increased in the $30 \mathrm{~m}$ period in both G1S and G1F.

Concerning spectral parameters, the MF and HL ratio underwent sustained upward trends in G1S, revealing a shift of the EHG-burst spectral content towards higher frequencies as the recording advanced. Being this effect not noted in G1F. The UAI parameter also exhibited an upward tendency in G1S with no distinguishing evolution in G1F. Teager energy values were higher in G1S (values from $0.69 \pm 0.75 \mu \mathrm{V}^{2} \cdot \mathrm{Hz}^{2} \cdot 10^{5}$ at basal to $1.43 \pm 1.45 \mu \mathrm{V}^{2} \cdot \mathrm{Hz}^{2} \cdot 10^{5}$ at the last analysis window) than G1F (values from $0.36 \pm 0.23 \mu \mathrm{V}^{2} \cdot \mathrm{Hz}^{2} \cdot 10^{5}$ at basal to $0.40 \pm 0.26 \mu \mathrm{V}^{2} \cdot \mathrm{Hz}^{2} \cdot 10^{5}$ at the last analysis window) in all the analysis blocks, with a tendency to rise in G1S and remain almost constant in G1F, as shown in figure 4.

The sample entropy declined throughout the recording session for both G1S and G1F, while Lempel-Ziv complexity only dropped slightly in G1S and remained almost constant in G1F. This indicates that as induction progresses Misoprostol produces more organized EHG-bursts than those in the basal period.

The EHG parameters' statistical significance $(\alpha=0.05)$ when comparing values in each 30 ' period with those of the basal period are shown in Figure 4. Sustained statistically significant changes, from 90 minutes (or earlier) until the end of the recording session, were obtained for peak-to-peak 
2 differences from 150 minutes until the end of the recording session.

3 Concerning the statistical test results for successful and failure groups, in Figure 4, it can be 4 observed that the peak-to-peak amplitude and Teager parameters are significantly higher for G1S than for G1F (shaded triangle) in more than one analysis window. In the case of Teager, these differences were sustained from 90 minutes until the end of the recording session. Teager is the most promising parameter to predict the success or failure of labor induction, although further studies are needed to corroborate these results.

\subsection{Uterine myoelectrical response to Dinoprostone induction drug}

Figure 5 shows mean values of the computed G2 parameters. Clear trends for G2S are distinguishable in duration (downward), peak-to-peak amplitude (upward) and NCT parameters (upward). EHG-burst duration was slightly reduced in the course of the recording session for G2S, while G2F exhibited a more erratic trend. Peak-to-peak amplitude in G2S increased and G2F also increased after the onset of labor induction showing mean values higher than those for G2S.

The spectral parameters related to the uterine myoelectrical response to Dinoprostone, the MF and HL ratios, presented slightly higher values for G2S than G2F and both spectral parameters showed a very slight upward tendency. Concerning UAI in G2S group, it gradually increased during induction, as did G2F, but more erratically, probably due to the small number of cases involved. The mean Teager energy values rose in both G2S and G2F, which could have been mainly due to the rise in EHG-burst amplitude, which was more marked in the G2F group.

As regards the complexity parameters, sample entropy slightly decreased in G2S and G2F during the first 4 hours of induction, while Lempel-Ziv complexity also marginally diminished in G2S, and was somewhat erratic in G2F.

Significant sustained changes (see Figure 5) of the values in each 30' period with respect to basal were obtained in G2S only for duration, peak to peak amplitude, NCT and the UAI EHG parameters. However, groups G2S and G2F showed similar trends in the first 4 h of induction and none of the parameters showed sustained significant difference between the success and failure groups. These results therefore suggest that the evolution of the EHG parameters during the first $4 \mathrm{~h}$ of induction may not be a good indicator of success when labor is induced with Dinoprostone.

\subsection{Uterine myoelectrical response:Misoprostol vs Dinoprostone}

In order to compare the electrophysiological response of both drugs, the temporal evolutions of the parameters extracted for G1S and G2S throughout the first 4 hours of induction are depicted in Figure 6. In both groups duration and peak-to-peak amplitude values decreased and increased, respectively. Nonetheless, in the Misoprostol group, amplitude gradually increased at the onset of labor induction, followed by a plateau after 150 minutes. The amplitude of the successful Dinoprostone group increased shortly after drug administration, presented a plateau from 60 to 150 minutes and then rose until the end of recording. NCT also showed increasing trends in both groups throughout the recording session, with mean values slightly higher for G2S than G1S. 
With reference to the spectral parameters, pronounced upward trends were observed in the G1S MF and HL ratio, with slightly higher mean values in the Misoprostol group. Indeed, MF exhibited statistically significant differences between G1S and G2S 4 hours after induction onset (Figure 6). UAI increased in both G1S and G2S, but G1S showed a higher growth rate $2 \mathrm{~h}$ after drug administration. Similarly, G1S presented higher values for Teager energy than G2S from 90 minutes after induction onset until the end of the recording session, with similar trends to those of amplitude in both groups. In the complexity parameters, sample entropy showed a downward tendency for both the G1S and G2S groups, a steeper slope in G1S than G2S and LZ exhibited an apparent downward trend only for G1S.

\section{Discussion}

Induced labor is associated with longer hospital stays and a higher caesarean rate than spontaneous labor and therefore needs more resources, due to more complications, such as uterine hyperstimulation and fetal heart rate anomalies, which could lead to a caesarean section [37]. Therefore, predicting the success of labor induction in the early stages is a key aspect in planning better labors in order to reduce maternal-fetal risks and healthcare costs. Obstetric parameters have been previously used to predict labor induction success, including maternal-fetal characteristics and cervical status such as maternal age, height, weight, birth weight, cervical length and Bishop score, among others [6-8], although not with a high degree of accuracy. Several studies have indicated that EHG could be useful in identifying the efficiency of uterine contractions due to the fact that term or preterm labor EHG-bursts differ from non-true labor bursts [19,20,28,38]. However, few efforts have been made to analyze the uterus' electrophysiological response to drugs during labor induction and none at all in predicting its success using spectral and complexity parameters extracted from the EHG-burst. In the present work, the myoelectrical response of the uterus to two commonly used cervical ripening drugs was analyzed to explore the possibility of predicting labor induction success in the first 4 hours by EHG parameters. For this, EHG recordings were made during induction and a series of temporal, spectral and complexity parameters were computed to determine those parameters that could be useful for predicting induction success.

Firstly, our results revealed that, with Misoprostol as the labor induction drug, differences in the uterine myoelectrical response of successful and unsuccessful inductions can be identified in the first 4 hours of induction. These differences were shown not only by the increased EHG-burst amplitude and number of contractions (EHG-burst) with respect to basal activity in successful inductions, but also a shift of the spectral content toward higher frequencies. This was not observed in failed inductions. They could thus be used as input parameters (alone or combined with traditional obstetric parameters) to design a predictor system of success or failure in the early stages of induction. These increases in temporal and spectral parameters are consistent with other studies that compared the features of EHG-bursts during pregnancy to those in the active phase of labor $[11,38,39]$. Moreover the increases were significant and sustained after 60-90 minutes of drug administration. These results are in agreement with Arronson et al [40], in which the effect of Misoprostol on uterine contractility was analyzed by calculating the Montevideo units from pressure recordings. Their results showed that after 1-2 h, regular uterine contractions appear and last at least up to $4 \mathrm{~h}$ after vaginal administration. The results are also consistent with pharmacokinetic studies on Misoprostol by other authors, who found that Misoprostol plasma 
concentration gradually increases after $400 \mu \mathrm{g}$ administered vaginally, reaching a peak between 75 and 80 minutes and then gradually drops until $6 \mathrm{~h}$ [41]. With reference to the complexity parameters, the decrease of the sample entropy values during the first 4 hours suggests that EHGbursts become more regular as labor induction progresses. This result is consistent with other studies that assessed EHG complexity in other obstetrical contexts. Vhrovec et al [42] found that sample entropy of the EHG signal decreased from the latent labor phase to the active phase, suggesting a reduction of signal complexity. The average sample entropy values of the whole EHG signal for term and pre-term delivery records drop as gestation progresses [28]. As regards LempelZiv, only G1S showed a non-erratic downward tendency. Nevertheless, there is no clear contribution of complexity parameters to forecast labor induction success or failure in the early stages of induction. In order to differentiate success and failed inductions when using Misoprostol, peak to peak amplitude, NCT and UAI showed statistical differences in at least one analysis window and only Teager presented sustained statistical differences. Nonetheless, the failed induction group is small. A more comprehensive database may reduce the variability of the results and sustained significant differences could be found in more EHG parameters.

In the successful Dinoprostone inductions, the duration, peak-to-peak amplitude, UAI and NCT showed clear trends throughout the recording session with sustained statistically significant differences with the basal period 60-120' after Dinoprostone administration. This result is consistent with a pharmacokinetic study that found that the peak plasma level was achieved between 60-120 minutes and the mean time to obtain sustained uterine activity was 127 minutes after vaginal Dinoprostone administration [43]. Nevertheless, the parameters that evolved during the first $4 \mathrm{~h}$ of induction did not show sustained statistical differences between G2S and G2F, with similar trends in both groups. A previous study found that during the first two hours after Dinoprostone administration, EMG activity did not change significantly and the maximum activity occurred between 2 and $8 \mathrm{~h}$ after this time [21]. This may explain why in the present work, the EHG-burst spectral parameters in women induced with Dinoprostone did not show sustained significant differences during the recording period (4h after administration). It would therefore seem necessary to extend the recording time to better analyze the electrophysiological response of the uterus to this drug.

When comparing uterine myoelectrical response in the first four hours for successful Misoprostol and Dinoprostone administration, it has been observed that this response differs according to the drug administered. Increasing spectral and time-frequency parameters trends and decreasing trends in regularity parameters were more notable and occured earlier for Misoprostol. These results suggest that using Misoprostol as a uterine stimulation agent increases the gap junction faster than Dinoprostone, and therefore the number of active cells during the EHG-burst, as well as increasing the ratio of the cells excitability [38], giving rise to effective contractions in a shorter time period. This may be due to Dinoprostone's dynamics being slower than Misoprostol, as has been found in several pharmacokinetics studies on these drugs [41,43]. It is also noteworthy that the Misoprostol group required a shorter time to achieve active labor than the Dinoprostone group $(15.8 \mathrm{~h}$ and 18.0 $\mathrm{h}$ for G1 and G2 respectively), even when the cervix state of the subjects induced with Misoprostol was more unfavorable (Bishop score: $1.4 \pm 1.1$ for G1 group vs. $2.2 \pm 0.9$ for G2 group). 
To sum up, the traditional uterine dynamics monitoring parameters such as NCT and duration are not by themselves able to determine the success of labor induction. Our results suggest that the combination of both the shift in the spectral content and the increment in the signal amplitude e.g. Teager energy, could be useful to identify successful inductions from a pharmacological point of view. In this regard, the relatively slow Dinoprostone dynamics needs a longer recording time to detect changes in the parameters to distinguish between success and failure. Nonetheless, a larger database, especially with a higher number of failed induction records than in the present study, is required to corroborate these results. In this context, it could be necessary to implement tools such as SMOTE and ADASYN to deal with the unbalanced data problem. Implementing predictor systems with different classification techniques and choosing the best prediction system would then be the next step in EHG labor induction studies.

It would also be interesting to study the capability of differentiating labor induction outcomes, i.e. vaginal deliveries $v s$ caesarean deliveries. In this scenario, there are external factors unrelated to uterine contractile activity that could lead to a caesarean section, such as loss of maternal-fetal wellbeing or pelvic-fetal disproportion. The EHG parameters would thus not be able to predict labor induction outcomes on their own. In this matter, an expert system would need to be developed that combines both obstetric and EHG information.

\section{Conclusions}

The uterine myoelectrical response of mothers induced with Misoprostol and Dinoprostone was analyzed and compared by calculating the temporal, spectral and complexity features of EHGbursts. Successful inductions by Misoprostol were associated with earlier effective contractions, with the EHG-bursts showing a remarkable increase of amplitude and shift of their spectral content towards higher frequencies at about 60-120 minutes after administration. In the subjects administered Misoprostol, the following parameters exhibited the different behavior of successful and failed inductions and could potentially be used for predicting success: peak-to-peak amplitude, MF, UAI and Teager. This latter also presented significant and sustained differences between success and failed inductions. With Dinoprostone, the parameters that showed sustained changes 4 $\mathrm{h}$ after induction (duration, NCT and UAI) could not differentiate between successful and failed inductions due to the slower Dinoprostone pharmacokinetics. A longer recording time would therefore be required for mothers given this drug in order to observe changes in the EHG-burst characteristics. Finally, these results indicate that it could be possible to use EHG parameters for prediction purposes and suggest that they could provide other valuable information on the myoelectrical state of the uterus during labor induction.

\section{Funding}

This research project was supported by the Spanish Ministry of Economy and Competitiveness, the European Regional Development Fund (DPI2015-68397-R) and the Bial business company

\section{References}

[1] Hamilton B, Osterman JK, Curtin S, Mathews T. Births: Final data for 2012. Hyattsville: 2012. 
[2] Filho OBM, Albuquerque RM, Cecatti JG. A randomized controlled trial comparing vaginal misoprostol versus Foley catheter plus oxytocin for labor induction. Acta Obstet Gynecol Scand 2010;89:1045-52. doi:10.3109/00016349.2010.499447.

[3] Seyb ST, Berka RJ, Socol ML, Dooley SL. Risk of cesarean delivery with elective induction of labor at term in nulliparous women. Obstet Gynecol 1999;94:600-7.

[4] Bishop EH. Pelvic Scoring For Elective Induction. Obstet Gynecol 1964;24:266-8.

[5] Faltin-Traub EF, Boulvain M, Faltin DL, Extermann P, Irion O. Reliability of the Bishop

[6] Crane JMG, Delaney T, Butt KD, Bennett KA, Hutchens D, Young DC. Predictors of successful labor induction with oral or vaginal misoprostol. J Matern Neonatal Med J 2004;15:319-23. doi:10.1080/14767050410001702195. score before labour induction at term. Eur J Obstet Gynecol Reprod Biol 2004;112:178-81. doi:10.1016/S0301-2115(03)00336-1.

[8] Pitarello P da RP, Tadashi Yoshizaki C, Ruano R, Zugaib M. Prediction of successful labor induction using transvaginal sonographic cervical measurements. J Clin Ultrasound 2013;41:76-83. doi:10.1002/jcu.21929.

[10] Catherine Tolcher M, Holbert MR, Weaver AL, McGree ME, Olson JE, El-Nashar SA, et al. Predicting Cesarean Delivery After Induction of Labor Among Nulliparous Women at Term. Obs Gynecol 2015;126:1059-68. doi:10.1097/AOG.0000000000001083.

[11] Vinken MPGC, Rabotti C, Mischi M, Oei SG. Accuracy of frequency-related parameters of the electrohysterogram for predicting preterm delivery: a review of the literature. Obstet Gynecol Surv 2009;64:529-41. doi:10.1097/OGX.0b013e3181a8c6b1.

[12] Euliano TY, Nguyen MT, Darmanjian S, McGorray SP, Euliano N, Onkala A, et al. Monitoring uterine activity during labor: a comparison of 3 methods. Am J Obstet Gynecol 2013;208:66.e1-66.e6. doi:10.1016/j.ajog.2012.10.873.

[13] Schlembach D, Maner WL, Garfield RE, Maul H. Monitoring the progress of pregnancy and labor using electromyography. Eur J Obstet Gynecol Reprod Biol 2009;144:S33-9. doi:10.1016/j.ejogrb.2009.02.016. pressure estimation using the Teager energy operator on electrohysterographic recordings. Physiol Meas Phys Eng Med Physiol Meas Physiol Meas 2014;35:1215-28. 
doi:10.1088/0967-3334/35/7/1215.

[16] Ye-Lin Y, Prats-Boluda G, Alberola-Rubio J, Bueno Barrachina J-M, Perales A, GarciaCasado J. Prediction of labor using non-invasive laplacian EHG recordings. 2013 35th Annu. Int. Conf. IEEE Eng. Med. Biol. Soc., vol. 2013, IEEE; 2013, p. 7428-31. doi:10.1109/EMBC.2013.6611275.

[17] Lucovnik M, Maner WL, Chambliss LR, Blumrick R, Balducci J, Novak-antolic Z, et al. Noninvasive Uterine Electromyography For Prediction of Preterm Delivery *. Am J Obs Gynecol 2011;3:228.e1-228.10. doi:10.1016/j.ajog.2010.09.024.

[18] Maner WL, Mackay LB, Saade GR, Garfield RE. Characterization of abdominally acquired uterine electrical signals in humans, using a non-linear analytic method n.d. doi:10.1007/s11517-005-0011-3.

[19] Fergus P, Cheung P, Hussain A, Al-Jumeily D, Dobbins C, Iram S. Prediction of Preterm Deliveries from EHG Signals Using Machine Learning. PLoS One 2013;8. doi:10.1371/journal.pone.0077154.

[20] Smrdel A, Jager F. Separating sets of term and pre-term uterine EMG records. Physiol Meas 2015;36:341-55. doi:10.1088/0967-3334/36/2/341.

[21] Aviram A, Melamed N, Hadar E, Raban O, Hiersch L, Yogev Y, et al. Effect of Prostaglandin E2 on Myometrial Electrical Activity in Women Undergoing Induction of Labor. J Perinatol 2014;31:413-8. doi:10.1055/s-0033-1352486.

[22] Tibor Toth. Transcutaneous Electromyography of Uterus in Prediction of Labor Outcome Induced by Oxytocine and Prostaglandine Shapes. Gynaecol Perinatol J Gynaecol Perinatol Reprod Med Ultrason Diagnostics 2005;14:75-6.

[23] Pandis GK, Papageorghiou AT, Ramanathan VG, Thompson MO, Nicolaides KH. Preinduction sonographic measurement of cervical length in the prediction of successful induction of labor. Ultrasound Obstet Gynecol 2001;18:623-8. doi:10.1046/j.09607692.2001.00580.x.

[24] Bueno B, San-Frutos L, Salazar F, Pérez-Medina T, Engels V, Archilla B, et al. Variables that predict the success of labor induction. Acta Obstet Gynecol Scand 2005;84:1093-7. doi:10.1111/j.0001-6349.2005.00881.x.

[25] Indraccolo U, Scutiero G, Greco P. Sonographic Cervical Shortening after Labor Induction is a Predictor of Vaginal Delivery. Rev Bras Ginecol E Obs 2016;38:585-8. doi:10.1055/s0036-1597629.

[26] Ware V, Raynor BD. Transvaginal ultrasonographic cervical measurement as a predictor of successful labor induction. Am J Obstet Gynecol 2000;182:1030-2. doi:10.1067/mob.2000.105399.

[27] Terrien J, Marque C, Karlsson B. Spectral characterization of human EHG frequency components based on the extraction and reconstruction of the ridges in the scalogram. 2007 29th Annu. Int. Conf. IEEE Eng. Med. Biol. Soc., IEEE; 2007, p. 1872-5. doi:10.1109/IEMBS.2007.4352680.

[28] Fele-Zorz G, Kavsek G, Novak-Antolic Z, Jager F. A comparison of various linear and nonlinear signal processing techniques to separate uterine EMG records of term and pre-term 
delivery groups. Med Biol Eng Comput 2008;46:911-22. doi:10.1007/s11517-008-0350-y.

[29] Terrien J, Marque C, Gondry J, Steingrimsdottir T, Karlsson B. Uterine electromyogram database and processing function interface: An open standard analysis platform for electrohysterogram signals. Comput Biol Med 2010;40:223-30. doi:10.1016/j.compbiomed.2009.11.019.

[30] Kaiser JF. On a simple algorithm to calculate the "energy" of a signal. Int. Conf. Acoust. Speech, Signal Process., IEEE; 1990, p. 381-4. doi:10.1109/ICASSP.1990.115702.

[31] Marque C, Duchene JMG, Leclercq S, Panczer GS, Chaumont J. Uterine EHG Processing for Obstetrical Monitorng. IEEE Trans Biomed Eng 1986;BME-33:1182-7. doi:10.1109/TBME.1986.325698.

[32] Hassan M, Terrien J, Alexandersson A, Marque C, Karlsson B. Nonlinearity of EHG signals used to distinguish active labor from normal pregnancy contractions. 2010 Annu. Int. Conf. IEEE Eng. Med. Biol., IEEE; 2010, p. 2387-90. doi:10.1109/IEMBS.2010.5627413.

[33] Radomski D, Grzanka A, Graczyk S, Przelaskowski A. Assessment of Uterine Contractile Activity during a Pregnancy Based on a Nonlinear Analysis of the Uterine Electromyographic Signal. Inf. Technol. Biomed., Berlin, Heidelberg: Springer Berlin Heidelberg; 2008, p. 325-31. doi:10.1007/978-3-540-68168-7_37.

[34] Vrhovec J, Lebar AM. An Uterine Electromyographic Activity as a Measure of Labor Progression. Appl. EMG Clin. Sport. Med. Dr. Catriona Steele, 2012. doi:10.5772/25526.

[35] Alamedine D, Diab A, Muszynski C, Karlsson B, Khalil M, Marque C. Selection algorithm for parameters to characterize uterine EHG signals for the detection of preterm labor. Signal, Image Video Process 2014;8:1169-78. doi:10.1007/s11760-014-0655-2.

[36] Lempel A, Ziv J. On the Complexity of Finite Sequences. IEEE Trans Inf Theory 1976;22:75-81. doi:10.1109/TIT.1976.1055501.

[37] Nicholson G, Cyr PL. Cost Of Failed Labor Induction: A Us Hospital Perspective. Value Heal 2013;16:A75. doi:10.1016/j.jval.2013.03.339.

[38] Garfield RE, Maner WL. Physiology and electrical activity of uterine contractions. Semin Cell Dev Biol 2007;18:289-95. doi:10.1016/j.semcdb.2007.05.004.

[39] Maner WL, Garfield RE, Maul H, Olson G, Saade G. Predicting term and preterm delivery with transabdominal uterine electromyography. Obstet Gynecol 2003;101:1254-60. doi:10.1016/S0029-7844(03)00341-7.

[40] Aronsson A, Bygdeman M, Gemzell-Danielsson K. Effects of misoprostol on uterine contractility following different routes of administration. Hum Reprod 2004;19:81-4. doi:10.1093/humrep/deh005.

[41] Shan Tang O, Schweer H, Seyberth HW, Lee SWH, Ho PC. Pharmacokinetics of different routes of administration of misoprostol. Hum Reprod 2002;17:332-6.

[42] Vrhovec J, Macek-Lebar A, Rudel D. Evaluating Uterine Electrohysterogram with Entropy. 11th Mediterr. Conf. Med. Biomed. Eng. Comput. 2007, Berlin, Heidelberg: 2007, p. 144-7. doi:10.1007/978-3-540-73044-6_36. 
1 [43] Yount SM, Lassiter N. The Pharmacology of Prostaglandins for Induction of Labor. J

2 Midwifery Women's Heal 2013;58:133-44. doi:10.1111/jmwh.12022.

3

4 